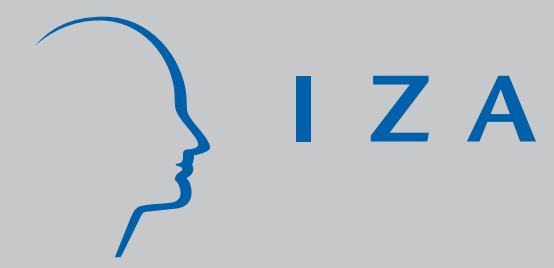

IZA DP No. 800

Sind Nominallöhne starr? Neuere Evidenz und wirtschaftspolitische Implikationen

Thomas Beissinger

Christoph Knoppik

J une 2003 


\title{
Sind Nominallöhne starr? Neuere Evidenz und wirtschaftspolitische Implikationen
}

\author{
Thomas Beissinger \\ University of Kaiserslautern, University of Regensburg \\ and IZA Bonn \\ Christoph Knoppik \\ University of Regensburg
}

Discussion Paper No. 800
June 2003

IZA

P.O. Box 7240

D-53072 Bonn

Germany

Tel.: +49-228-3894-0

Fax: +49-228-3894-210

Email: iza@iza.org

This Discussion Paper is issued within the framework of IZA's research area General Labor Economics. Any opinions expressed here are those of the author(s) and not those of the institute. Research disseminated by IZA may include views on policy, but the institute itself takes no institutional policy positions.

The Institute for the Study of Labor (IZA) in Bonn is a local and virtual international research center and a place of communication between science, politics and business. IZA is an independent, nonprofit limited liability company (Gesellschaft mit beschränkter Haftung) supported by Deutsche Post World Net. The center is associated with the University of Bonn and offers a stimulating research environment through its research networks, research support, and visitors and doctoral programs. IZA engages in (i) original and internationally competitive research in all fields of labor economics, (ii) development of policy concepts, and (iii) dissemination of research results and concepts to the interested public. The current research program deals with (1) mobility and flexibility of labor, (2) internationalization of labor markets, (3) welfare state and labor market, (4) labor markets in transition countries, (5) the future of labor, (6) evaluation of labor market policies and projects and (7) general labor economics.

IZA Discussion Papers often represent preliminary work and are circulated to encourage discussion. Citation of such a paper should account for its provisional character. A revised version may be available on the IZA website (www.iza.org) or directly from the author. 


\title{
ABSTRACT
}

\section{Sind Nominallöhne starr? \\ Neuere Evidenz und wirtschaftspolitische Implikationen*}

\begin{abstract}
Bei Vorliegen nach unten starrer Nominallöhne erschwert niedrige Inflation Reallohnanpassungen und führt so möglicherweise zu erhöhter gleichgewichtiger Arbeitslosigkeit. Dieser Aufsatz analysiert die wachsende Evidenz zu nach unten starren Nominallöhnen. Es werden nicht nur die Einschätzungen der Existenz und des Ausmaßes der Nominallohnrigidität diskutiert, sondern auch die möglichen Erklärungen für derartige Rigidität sowie ihre makroökonomischen Auswirkungen. Wir kommen zu dem Schluss, dass Abwärtsnominallohnstarrheit existiert, von erheblichem Ausmaß ist und dass aller Wahrscheinlichkeit nach ihre realen Auswirkungen bei sehr niedriger Inflation nicht zu vernachlässigen sind. Die Ergebnisse dieser Literatur sollten daher bei der Formulierung von Inflationszielen Berücksichtigung finden.
\end{abstract}

\section{Are Nominal Wages Rigid? Recent Evidence and Policy Implications}

In the presence of downwardly rigid nominal wages, low inflation may lead to higher equilibrium unemployment by hindering real wage adjustments. This paper surveys and analyses the growing evidence on downwardly rigid nominal wages. We deal not only with assessments of existence and extent of downward nominal wage rigidity, but also discuss its possible explanations and macroeconomic consequences. We conclude that downward nominal wage rigidity does exist, that its extent is substantial and that very likely the real consequences at low, close-to-zero inflation are non-negligible. The results of this strand of research should therefore be taken into account in the formulation of optimal inflation targets.

JEL Classification: J30, E24, E31, E52

Keywords: nominal wage rigidity, equilibrium unemployment, Phillips Curve, inflation target, Germany

Corresponding author:

Thomas Beissinger

University of Regensburg

93040 Regensburg

Germany

Email: thomas.beissinger@wiwi.uni-regensburg.de

\footnotetext{
*Für hilfreiche Kommentare zu einer früheren Fassung danken wir Eva Ackstaller, Lutz Arnold, Kerstin Frings, Jürgen Heubes und Karola Schmitt.
} 


\section{Einleitung}

Seit etwas mehr als einem Jahrzehnt hat sich im Kreis der Zentralbanken der westlichen Industrieländer die Überzeugung durchgesetzt, dass es die vorrangige Aufgabe der Geldpolitik sei, für niedrige Inflationsraten zu sorgen. Diese Überzeugung wurde seitdem weitgehend erfolgreich in die Tat umgesetzt, zum Teil in bemerkenswertem Kontrast zu den früher verfolgten Politiken. Mit der Realisierung sehr niedriger Inflationsraten sind allerdings von wissenschaftlicher Seite auch Warnungen vor den möglichen Gefahren zu niedriger Inflation laut geworden. Eines der Risiken könnte in nach unten starren Nominallöhnen liegen, die in Verbindung mit niedriger Inflation notwendige Reallohnanpassungen behindern und so möglicherweise zu erhöhter gleichgewichtiger Arbeitslosigkeit führen. Zur Klärung dieses Sachverhalts hat sich im Laufe der 90er Jahre eine wissenschaftliche Debatte um Existenz und Ausmaß von Abwärtsnominallohnstarrheit entwickelt, deren Ergebnisse sich in der jüngeren wirtschaftspolitischen und öffentlichen Diskussion bei nationalen und internationalen Organisationen sowie in den Medien widerspiegeln. ${ }^{1}$

Ziel dieses Aufsatzes ist ein umfassender Überblick zu den Begründungen, der Existenz, dem Ausmaß, den realen Konsequenzen und den wirtschaftspolitischen Implikationen nach unten starrer Nominallöhne. Abschnitt 2 erläutert, wie aus theoretischer Sicht eine zu niedrige Inflation bei Abwärtsnominallohnstarrheit die Arbeitslosigkeit erhöhen kann. Bevor aber aufgrund dieses Arguments die Inflationsziele der Zentralbanken auf den Prüfstand gestellt werden, ist zunächst nachzuweisen, dass das Phänomen nach unten starrer Nominallöhne existiert und quantitativ bedeutsam ist. Implikationen für die Wirtschaftspolitik können zudem nur abgeleitet werden, wenn bekannt ist, auf welche Faktoren Abwärtsnominallohnstarrheit

1 Als Beispiele können die folgenden Veröffentlichungen dienen: IMF (1999), Economist (2000a), Economist (2000b), die Beiträge in Europäische Zentralbank (2001), OECD (2002), Economist (2002) und Europäische Zentralbank (2003) sowie die hierin zusammengefassten Beiträge. 
zurückzuführen ist. Möglicherweise verschwindet das Phänomen rigider Löhne in einer Umwelt mit niedriger Inflation von selbst oder kann durch geeignete Arbeitsmarktreformen beseitigt werden, so dass ein Handeln der Geldpolitik nicht geboten ist. Abschnitt 3 liefert erste Hinweise für die Existenz nach unten starrer Nominallöhne und führt mögliche Begründungen für dieses Phänomen an. Abschnitt 4 diskutiert die mikroökonometrische Evidenz zu Existenz und Ausmaß von Abwärtsnominallohnstarrheit. Abschnitt 5 präsentiert eine Abschätzung der makroökonomischen Konsequenzen, die sich aus der empirischen Evidenz ergeben. Insbesondere wird untersucht, mit welchem Anstieg der Arbeitslosigkeit bei niedriger Inflation aufgrund von Nominallohnstarrheit zu rechnen ist. Abschnitt 6 bewertet die präsentierte Evidenz und erläutert die wirtschaftspolitischen Implikationen.

\section{Inflation und Arbeitslosigkeit - Kein Langfrist-trade-off, oder doch?}

Seit Friedman (1968) und Phelps (1967) teilen Ökonomen mehrheitlich die Auffassung, dass zwischen Inflation und Arbeitslosigkeit auf Dauer kein Zusammenhang besteht. Diese These steht nicht im Widerspruch zur Beobachtung, dass es aufgrund von Konjunkturschwankungen kurzfristig einen negativen Zusammenhang von Inflation und Arbeitslosigkeit geben kann. Im Regelfall geht die Arbeitslosigkeit im Aufschwung bei gleichzeitig steigender Inflation zurück, während im Abschwung der Anstieg der Arbeitslosigkeit von einem Rückgang der Inflation begleitet wird.

In einer langfristigen Betrachtung, die von den Schwankungen der ökonomischen Aktivität im Konjunkturzyklus abstrahiert, liegt jedoch eine Arbeitslosenquote $u^{*}$ vor, die von der Höhe der Inflation unbeeinflusst ist und alleine von strukturellen Faktoren abhängt, wie z. B. der Höhe der Arbeitslosenunterstützung und der Lohnnebenkosten. Bei dieser gleichgewichtigen Arbeitslosenquote geht vom Arbeitsmarkt kein Inflationsdruck aus, d. h. $u^{*}$ ist 
mit konstanter Inflation vereinbar. Für $u^{*}$ hat sich deshalb auch das Akronym NAIRU (NonAccelerating-Inflation Rate of Unemployment) eingebürgert. ${ }^{2}$

Eine graphische Darstellung des Zusammenhangs zwischen Inflation und Arbeitslosigkeit wird als Phillipskurve bezeichnet. Da es nach gängiger Meinung auf lange Sicht keinen Zusammenhang zwischen Inflation und Arbeitslosigkeit gibt, sollte die langfristige Phillipskurve senkrecht sein (siehe Abbildung 1a). Die Zentralbank kann daher mit ihrer Geldpolitik eine als optimal erachtete Inflationsrate (z. B. Punkt A oder Punkt B) wählen, ohne damit die NAIRU $u^{*} \mathrm{zu}$ beeinflussen.

\section{Abbildung 1}

Als prominenter Kritiker an dieser Sichtweise wird üblicherweise James Tobin ins Feld geführt. Tobin (1972) argumentierte, dass eine zu geringe Inflationsrate die Arbeitslosigkeit erhöhen könnte, falls die Nominallöhne nach unten starr sind, d. h. falls die Nominallöhne nicht gesenkt werden können, aus welchen Gründen auch immer. Tobins Hypothese, dass eine bestimmte Mindesthöhe der Inflation von Vorteil sei (,inflation greases the wheels of the labor market”), spielte in der wissenschaftlichen Debatte der beiden nachfolgenden Jahrzehnte keine große Rolle mehr. Dies ist zum einen auf die hohen Inflationsraten in den 70er und 80er Jahren zurückzuführen, die das (behauptete) Problem einer zu niedrigen Inflationsrate eher als ein theoretisches Kuriosum erscheinen ließen. Zum anderen deutet die These von nach unten starren Nominallöhnen auf die Existenz von Geldillusion hin und scheint damit ein irrationales Verhalten der Wirtschaftssubjekte zu implizieren. Geldillusion liegt vor, wenn Wirtschaftsubjekte ihre Entscheidungen und ihr Verhalten an nominellen statt an realen Größen festmachen. Das Marktverhalten rational handelnder Wirtschaftssubjekte sollte hingegen ausschließlich von realen Größen (z. B. relativen Preisen) und nicht von nominellen

\footnotetext{
2 Siehe beispielsweise Layard, Nickell und Jackman (1991) und Landmann und Jerger (1999).
} 
Größen (z. B. Preisen in Geldeinheiten) abhängen. Die Geldillusionsthese widerspricht also dem auch in makroökonomischen Modellen üblicherweise vertretenen Postulat rationalen Verhaltens.

Seit den 90er Jahren sind jedoch die Inflationsraten in den meisten industrialisierten Ländern deutlich zurückgegangen. Zudem steigt die Bereitschaft, die ökonomischen Implikationen eines vom strikten Rationalitätspostulat abweichenden Verhaltens zu untersuchen oder mögliche rationale Begründungen für ein zunächst als irrational betrachtetes Verhalten von Wirtschaftssubjekten zu finden. Diese Entwicklungen haben den Boden für die inzwischen klassische Arbeit von Akerlof, Dickens und Perry (1996) bereitet, die der Forschung zu den Konsequenzen nach unten starrer Nominallöhne in den vergangenen Jahren einen enormen Auftrieb verlieh. Wichtigstes Ergebnis der Studie von Akerlof, Dickens und Perry ist die Erkenntnis, dass die langfristige Phillipskurve für niedrige Inflationsraten nicht vertikal verläuft, sondern geneigt ist. In Abbildung $1 \mathrm{~b}$ ist ersichtlich, dass für unterschiedlich hohe Inflationsraten unterschiedlich hohe Werte für die gleichgewichtige Arbeitslosenquote resultieren. In Punkt $A$ ist die Inflationsrate hinreichend groß, um zur minimalen gleichgewichtigen Arbeitslosenquote $u_{\min }^{*} \mathrm{zu}$ führen; diese entspricht dem Wert $u^{*}$ aus Abbildung 1a. Ist die Inflationsrate jedoch beispielsweise auf dem niedrigeren Niveau des Punktes B, so ist das aktuelle Niveau der NAIRU $u_{B}^{*}$ höher als die minimale gleichgewichtige Arbeitslosenquote. Das Zusammenspiel von Niedriginflation und nach unten starren Nominallöhnen führt daher im Beispiel zur Zusatz-Arbeitslosigkeit (excess unemployment) in Höhe von $u_{B}^{*}-u_{\min }^{*}$. Den Simulations- und Schätzergebnissen von Akerlof, Dickens und Perry (1996) zufolge kann die Zusatz-Arbeitslosigkeit bei Nullinflation sogar mehrere Prozentpunkte betragen. Dagegen würde bereits eine Inflationsrate von etwa drei Prozent ausreichen, um die Realisation der minimalen Arbeitslosenquote $u_{\min }{ }^{*}$ zu gewährleisten. 
Um diese Ergebnisse nachvollziehen zu können, müssen die Prozesse auf dem Arbeitsmarkt eingehender betrachtet werden. Akerlof, Dickens und Perry (1996) gehen von der realistischen Annahme aus, dass Güter- und Arbeitsmärkte durch unvollkommenen Wettbewerb charakterisiert sind und Firmen und Gewerkschaften heterogen sind. So produzieren die Firmen zum Beispiel unterschiedliche Güter und sehen sich daher unterschiedlichen Nachfragebedingungen ausgesetzt. Aufgrund ihrer Marktmacht verfügen Firmen und Gewerkschaften über Preis- und Lohnsetzungsspielräume, die in Verbindung mit der angenommenen Heterogenität zu unterschiedlichen Reallöhnen in den einzelnen Firmen führen. Gleichgewichtssituationen in dieser Ökonomie sind alles andere als statisch, sondern vielmehr von ständigen, heterogenen Angebots- und Nachfragestörungen geprägt. So gibt es auch im Gleichgewicht beispielsweise immer Firmen, deren Güternachfrage ansteigt, während andere Firmen aufgrund von Nachfragerückgängen in Schwierigkeiten geraten. Bei unvollständigem Wettbewerb reagieren Firmen auf Güternachfrageänderungen mit Änderungen der Preise, der Produktion und der Arbeitsnachfrage.

Nun sei eine einzelne Firma betrachtet, die auf einen Rückgang der Güternachfrage unter anderem mit einer Verringerung der Arbeitsnachfrage reagiert. Bleiben die Reallöhne unverändert, so erfolgt eine Reduktion der Belegschaft. Werden die Reallöhne hingegen gesenkt, so kann der Beschäftigungsabbau verringert oder gänzlich vermieden werden. In einer Umwelt mit vergleichsweise hoher Inflation können die Reallöhne einfach dadurch gesenkt werden, dass die Nominallöhne mit geringerer Rate als die Preise wachsen. Bei einer Inflationsrate von fünf Prozent kann beispielsweise eine Reduktion der Reallöhne um drei Prozent einfach dadurch herbeigeführt werden, dass die Nominallöhne nur um zwei Prozent erhöht werden. Die Tatsache, dass es mögliche Widerstände gegen eine Senkung der Nominallöhne gibt, ist in diesem Szenario nicht von Belang, da die Nominallöhne sogar erhöht werden. In einer Ökonomie mit geringen Inflationsraten führt die Existenz nach unten 
starrer Nominallöhne dagegen zu realwirtschaftlichen Konsequenzen. Beträgt beispielsweise die Inflationsrate nur ein Prozent, so kann eine Reallohnsenkung von drei Prozent nur durch eine zweiprozentige Nominallohnsenkung herbeigeführt werden. Gibt es Widerstände gegen eine Reduktion der Nominallöhne, so können die Reallöhne nicht im erforderlichen Ausmaß gesenkt werden. Die entsprechende Firma wird dann mit einer stärkeren Reduktion der Beschäftigung reagieren als bei Nominallohnflexibilität.

Für die gesamte Ökonomie führt das Zusammenwirken von niedriger Inflation und nach unten starren Nominallöhnen dazu, dass ein größerer Teil der Firmen mit ungünstiger Nachfrageentwicklung zu einer Beschäftigungsreduktion gezwungen ist, als dies entweder bei Nominallohnflexibilität oder bei höherer Inflation der Fall wäre. Im Ergebnis ist bei Vorliegen von Nominallohnstarrheit die aggregierte Arbeitslosenquote bei niedriger Inflation höher als bei höheren Inflationsraten, was den geneigten Verlauf der langfristigen Phillipskurve bei niedrigen Inflationsraten erklärt.

\section{Begründungen für Nominallohnstarrheit}

Die Existenz nach unten starrer Nominallöhne wird in der Literatur vor allem auf psychologisch begründete Verhaltensmuster und auf institutionelle Regelungen zurückgeführt. Diese beiden Erklärungsansätze scheinen auf den ersten Blick sehr gegensätzlich zu sein. Die Diskussion in diesem Abschnitt legt jedoch die Vermutung nahe, dass die beiden Erklärungen zueinander komplementär sind.

\subsection{Psychologische Ansätze}

Psychologische Ansätze beruhen üblicherweise auf der These eines durch Fairnessüberlegungen beeinflussten Verhaltens in Kombination mit der Existenz von Geldillusion. Derartige 
Ansätze werden von Ökonomen allerdings nur zögerlich (wenn überhaupt) akzeptiert, da sie mit dem ökonomischen Postulat der Abwesenheit von Geldillusion kollidieren.

Psychologische Studien legen jedoch nahe, dass Geldillusion ein bedeutendes und häufig auftretendes Phänomen darstellt; vgl. Shafir, Diamond und Tversky (1997). Ein wichtiges Ergebnis der kognitiven Psychologie ist die Erkenntnis, dass alternative Repräsentationen (sogenannte „Frames“) ein und derselben Situation das Verhalten der Individuen in diesen Situationen in systematischer Weise beeinflussen. Beispielsweise können ökonomische Transaktionen in nominellen Größen oder in realen Größen formuliert werden. Die Art der Darstellung beeinflusst die Bewertung dieser Transaktionen durch die Wirtschaftssubjekte.

Shafir, Diamond und Tversky (1997) erklären die Existenz von Geldillusion durch multiples Framing. Ökonomische Entscheidungssituationen im täglichen Leben werden mal mehr, mal weniger aus der Perspektive des realen oder nominellen Frames betrachtet, je nachdem, welcher Frame für die Entscheidungssituation geeigneter zu sein scheint. Bei nicht zu hohen Inflationsraten kann es einfacher und ausreichend akkurat sein, in nominellen Größen zu rechnen, obwohl man strenggenommen dabei Fehler macht.

Die Existenz und Bedeutung von Geldillusion ist auch durch die experimentelle Studie von Fehr und Tyran (2001) belegt. Die Autoren erhalten in ihren Experimenten deutliche Hinweise darauf, dass sich das Phänomen der Nominallohnstarrheit vor allem auf Lohnkürzungen, nicht jedoch auf Lohnerhöhungen erstreckt. Nominelle Rigiditäten werden zudem dadurch verstärkt, dass Individuen in ihren Entscheidungskalkülen davon ausgehen, dass sich andere Wirtschaftssubjekte in ihrem Verhalten von Geldillusionen leiten lassen und die Änderung nomineller Größen als Approximation für die Änderung realer Größen heranziehen. 
Nicht nur das Phänomen Geldillusion kollidiert mit dem von Ökonomen bevorzugten Menschenbild des homo oeconomicus. Reziproke Verhaltensmuster stehen ebenfalls im Gegensatz zur These des alleine auf den Eigennutz bedachten Individuums. Eine Vielzahl experimenteller Studien weist nach, dass Individuen beispielsweise auf ein unfair empfundenes Verhalten anderer Personen mit „Bestrafungsaktionen“ reagieren, selbst wenn ein derartiges reziprokes Verhalten nur Kosten, nicht jedoch (materielle) Erträge mit sich bringt, siehe Fehr und Gächter (2000). Diese Erkenntnisse sind für das Verständnis der Funktionsweise von Arbeitsmärkten von besonderer Relevanz. Arbeitsverträge sind üblicherweise unvollständige Verträge, d. h. in den Verträgen ist die Entlohnung festgesetzt, während die vom Arbeitnehmer zu erbringende Gegenleistung nicht in allen Details geregelt werden kann. Bestimmte Aspekte der Arbeitsleistung, wie beispielsweise der Grad an gezeigter Eigeninitiative, gutes Urteilsvermögen etc., lassen sich schlichtweg nicht vertraglich fixieren. Angesichts unvollständiger Arbeitsverträge ist es für den Arbeitgeber wichtig, eine kooperative Arbeitsatmosphäre zu schaffen und zu erhalten. Weisen die Arbeitnehmer reziproke Verhaltensmuster auf, so wird der Arbeitgeber vor Aktionen zurückschrecken, die von den Arbeitnehmern als unfair empfunden werden. Beispielsweise zahlen Firmen freiwillig Löhne, die über dem Anspruchslohn der Arbeitnehmer liegen. Sollten arbeitslose Personen anbieten, zu einem niedrigeren Lohn $\mathrm{zu}$ arbeiten, so werden derartige Lohnunterbietungen nicht akzeptiert, da dies von der aktuellen Belegschaft als unfair empfunden werden könnte und damit negative Auswirkungen auf die Arbeitsmoral hätte; siehe Fehr und Falk (1999).

Befragungsstudien, auf die weiter unten näher eingegangen wird, weisen nach, dass Arbeitgeber vor Nominallohnkürzungen zurückschrecken, da diese von Arbeitnehmern im Regelfall als unfair empfunden werden. Ausnahmen ergeben sich lediglich in Situationen, in denen Arbeitgeber glaubhaft nachweisen können, dass Nominallohnkürzungen für den Fortbestand der Firma unerlässlich sind. Es stellt sich natürlich die Frage, warum sich Arbeitnehmer 
in ihren Fairnessvorstellungen am Niveau der Nominallöhne und nicht am Niveau der Reallöhne orientieren sollten. Shafir, Diamond und Tversky (1997) zeigen jedoch, dass Geldillusion die Auffassungen der Arbeitnehmer über die gerechte Entlohnung beeinflusst. FairnessÜberlegungen werden oftmals auf Grundlage eines nominellen Frames vorgenommen. Eine Nominallohnkürzung um 2 Prozent in Zeiten mit Nullinflation wird daher in deutlich stärkerem Maße als unfair eingestuft als ein Nominallohnanstieg um 2 Prozent in Zeiten mit 4 Prozent Inflation, obwohl beide Situationen denselben Reallohnrückgang von 2 Prozent implizieren. Eine mögliche Begründung hierfür ist, „... price rises are much more impersonal and cannot be blamed on the workers' own employer“, Rees (1973), S. 227.

Zusammenfassend lässt sich also festhalten, dass psychologische Ansätze zur Erklärung nach unten starrer Nominallöhne auf der Kombination von Fairnessüberlegungen und der Existenz von Geldillusion beruhen.

\subsection{Institutionelle Begründungen}

Institutionelle Begründungen für nach unten starre Nominallöhne setzen beim Lohnverhandlungsprozess an, der je nach Land oder betrachtetem Wirtschaftszweig zwischen Arbeitgeber oder Arbeitgeberverband auf der einen Seite und Gewerkschaften oder dem einzelnen Arbeitnehmer auf der anderen Seite stattfindet. Zur Vereinfachung der Darstellung sei im folgenden angenommen, dass die Entlohnung zwischen einem Arbeitgeber und einer Gewerkschaft ausgehandelt wird. Die Änderung der im Arbeitsvertrag festgesetzten nominellen Entlohnung erfordert üblicherweise die beiderseitige Zustimmung von Arbeitgeber und Gewerkschaft. Selbst bei Ablauf des Tarifvertrags, der dem Arbeitsvertrag des einzelnen Arbeitnehmers zugrunde liegt, ist es für den Arbeitgeber oftmals nicht möglich, einseitig eine Nominallohnkürzung durchzusetzen. Im Regelfall wird die Produktion unter den Bedingungen des alten Arbeitsvertrags fortgesetzt, bis eine neue Einigung zustande kommt (Nachwirkung des 
Tarifvertrags). Bei Null-Inflation ergibt sich bei den alten Nominallöhnen hierbei keine Reallohnreduktion, bei höherer Inflation dagegen schon. Will sich daher die Gewerkschaft gewünschten Reallohnkürzungen widersetzen, so kann sie dies bei Null-Inflation wegen der Nachwirkung problemlos tun. Bei höherer Inflation wird sie dagegen gewisse Reallohnkürzungen unter Umständen hinnehmen, da das Weitergelten des alten Nominallohns ebenfalls mit Reallohneinbußen verbunden ist. Es lässt sich zeigen, dass die hieraus resultierende Nominallohnstarrheit umso stärker ist, je umfassender der Geltungsbereich von Tarifverträgen ist und umso strikter Kündigungsschutzregelungen sind, vgl. Holden (2002).

Der institutionelle Begründungsansatz führt zu einer Erklärung nach unten starrer Nominallöhne, die mit dem Rationalverhalten der Wirtschaftssubjekte vereinbar ist. Er lässt jedoch auch einige Fragen offen. Beispielsweise wird nicht erklärt, warum Arbeits- bzw. Tarifverträge eine nominelle Entlohnung festlegen und keine Indexierungsklauseln enthalten, die eine Anpassung an Änderungen des Preisniveaus ermöglichen würden.

Der hier beschriebene institutionelle Ansatz wird in der Literatur oftmals als Alternative zum psychologischen Ansatz zur Erklärung nach unten starrer Löhne angesehen. Es stellt sich allerdings die Frage, ob institutionelle Regelungen als exogen betrachtet werden sollten. Die Tatsache, dass das Arbeitsrecht unilateralen Lohnkürzungen in vielen Ländern einen Riegel vorschiebt, könnte selbst das Ergebnis von Fairnessüberlegungen sein, die ihren Niederschlag in entsprechenden institutionellen Regelungen gefunden haben. Umgekehrt ist natürlich auch nicht auszuschließen, dass institutionelle Regelungen die Auffassungen über Fairness beeinflussen. Psychologischer und institutioneller Ansatz sollten daher eher als komplementäre, anstatt als sich widersprechende Sichtweisen interpretiert werden, vgl. Holden (2002). 


\subsection{Befragungsstudien}

Einen Eindruck vom Ausmaß und den Ursachen der Nominallohnstarrheit in der betrieblichen Praxis erhält man durch Studien, in denen Firmen zu ihrer Lohnpolitik befragt werden (siehe Tabelle 1). In einigen Studien sollten Firmen Angaben über die in der Vergangenheit vorgenommenen Nominallohnkürzungen machen. Aus Spalte 4 von Tabelle 1 wird ersichtlich, dass die dokumentierte Nominallohnstarrheit mit einer Ausnahme sehr hoch ist, d. h. sich üblicherweise auf nahezu alle Firmen der Stichprobe erstreckt. Lediglich in der Studie von Blinder und Choi (1990), die allerdings nur 19 Firmen umfasst, nahm ein Viertel der Firmen vor allem wegen der schwachen Nachfrage nach ihren Produkten eine (zum Teil temporäre) Nominallohnkürzung vor.

Natürlich hängt die Häufigkeit von beobachteten Nominallohnkürzungen auch von der jeweiligen Höhe der Inflationsrate und der Rate des Produktivitätswachstums ab. Ist beispielsweise die Inflationsrate sehr hoch, ist es nicht weiter überraschend, wenn keine Nominallohnkürzungen beobachtet werden. Für eine Beurteilung der Relevanz von Nominallohnstarrheit muss daher eigentlich das wirtschaftliche Umfeld der jeweiligen Befragung berücksichtigt werden. ${ }^{3}$ Unter diesem Aspekt sind die Untersuchungen von Agell und Lundborg (2003) und Agell und Bennmarker (2002) von besonderem Interesse. Obwohl Schweden insbesondere in der zweiten Hälfte der neunziger Jahren eine extrem niedrige Inflation von durchschnittlich einem Prozent aufwies und obgleich die Arbeitslosigkeit sehr hoch war, finden diese Autoren keinerlei Anzeichen eines Rückgangs der nahezu vollständigen Nominallohnstarrheit nach unten. ${ }^{4}$

\section{Tabelle 1}

3 Eine detaillierte Beschreibung des wirtschaftlichen Umfelds, in dem die einzelnen Länderstudien durchgeführt wurden, würde den Rahmen dieses Aufsatzes sprengen. Als Anhaltspunkt ist in Spalte 3 das Jahr angegeben, in dem die Befragung durchgeführt wurde.

4 Zur Entwicklung von Inflation und Arbeitslosigkeit in den 90er Jahren in Schweden siehe Agell und Lundborg (2003), S. 16-17. 
In den in Tabelle 1 aufgeführten Studien wurden die Manager auch nach den Gründen für unterbliebene Nominallohnkürzungen gefragt. Aus den Antworten geht übereinstimmend die große Bedeutung von Fairnessüberlegungen als Begründung für nicht durchgeführte Lohnkürzungen hervor. Eine Reduktion der Entlohnung wird nur dann akzeptiert, wenn sie dazu dient, das Überleben einer in ökonomischer Schieflage befindlichen Firma zu sichern. Wird hingegen die Lohnpolitik der Firma von den Arbeitnehmern als unfair wahrgenommen, so befürchtet das Management eine höhere Fluktuation der Beschäftigten, einen Rückgang der Arbeitsanstrengungen sowie einen Rückgang der Qualität zukünftiger Arbeitsplatzbewerber. Werden anstelle einer Lohnanpassung Arbeitszeitreduktionen und selektive Entlassungen durchgeführt, so scheint die Arbeitsmoral der verbleibenden Belegschaft in geringerem Ausmaß negativ berührt zu sein. Wie bei der Diskussion des psychologischen Erklärungsansatzes ausgeführt, kann die Tatsache, dass Nominallohnkürzungen als unfair empfunden werden und die Effizienz beeinflussen, nur mit der Existenz von Geldillusion erklärt werden. In manchen Studien wie Blinder und Choi (1990) und Bewley (1999) wird die Rolle von Geldillusion bei der Frage der als fair betrachteten Entlohnung explizit bestätigt.

Aus den oben zitierten Studien für Schweden und der Arbeit von Franz und Pfeiffer (2003) für Deutschland wird außerdem deutlich, dass Nominallohnrigiditäten auch durch institutionelle Regelungen hervorgerufen bzw. verstärkt werden. Insbesondere arbeitsrechtliche und tarifvertragliche Regelungen erschweren die Senkung der Nominallöhne, wobei dieser Effekt der Studie von Franz und Pfeiffer zufolge vor allem für die gering qualifizierten Arbeitnehmer von Bedeutung ist. Betrachtet man die bisherige Evidenz aus Befragungsstudien, so wird ein Einfluss von Arbeitsmarktinstitutionen auf den Umfang der Lohnrigidität nur in den Ländern nachgewiesen, in denen Gewerkschaften, Tarifverträge etc. eine vergleichsweise große Rolle spielen. Diese Abgrenzung entspricht der üblichen Klassifizierung in kontinental-europäisch bzw. angelsächsisch geprägte Arbeitsmarktregimes. 
Obwohl die Befragungsstudien zu vielen nützlichen Erkenntnissen führen, stellt sich die Frage, ob die Ergebnisse überzeugend den Grad der Nominallohnrigidität in der jeweiligen Ökonomie zum Ausdruck bringen. Ein Problem ist, dass die meisten Studien nicht auf einem repräsentativen Firmensample beruhen. ${ }^{5}$ Oftmals werden nur sehr wenige Firmen oder ausgewählte Wirtschaftszweige betrachtet. In vielen Studien sind kleine Firmen nicht in repräsentativem Umfang vertreten. Aufgrund der teilweise geringen Rücklaufquoten besteht zudem die Gefahr einer Verzerrung der Ergebnisse. Ein weiteres Problem besteht darin, dass in Befragungsstudien möglicherweise bewusst falsche Antworten gegeben werden oder aber die Antworten durch die Art der Fragestellung in eine bestimmte Richtung beeinflusst werden. Befragungsstudien weisen außerdem den Nachteil auf, dass quantitative Schlussfolgerungen über das Ausmaß der Nominallohnrigidität in einer Ökonomie nur sehr eingeschränkt gezogen werden können.

Um diese Nachteile zu vermeiden, bietet sich die Analyse großer Mikrodatensätze an, die Informationen über das tatsächliche Marktverhalten der Wirtschaftssubjekte enthalten. Besonders geeignet scheinen Datenquellen, die Auskunft über die individuelle Entlohnung geben und repräsentativ für die Gesamtökonomie sind. Die verschiedenen Forschungsstrategien zur Identifizierung von Nominallohnrigiditäten in derartigen Mikrodatensätzen sind Gegenstand des nachfolgenden Abschnitts.

\section{Mikroökonometrische Evidenz zu Existenz und Ausmaß von Abwärtsno- minallohnstarrheit}

Die verwendeten Mikrodaten liefern in der Regel Informationen über Entgelte aus individuellen Arbeitsverhältnissen. Meist handelt es sich hierbei um Panels, d. h. für einen Querschnitt

5 Eine Ausnahme stellt die Studie von Agell und Bennmarker (2002) dar, die auf einer repräsentativen Zufallsstichprobe für verschiedene Wirtschaftsbereiche und Firmengrößen basiert. 
von Arbeitnehmern liegen Beobachtungen nicht nur für einen Zeitpunkt, sondern für mehrere Zeitpunkte vor. Typischerweise entstehen die Panel-Daten durch Befragungen von Arbeitnehmern; in einigen Fällen finden aber auch administrative Daten der Sozialversicherungen, Personaldaten von Unternehmen, Befragungsdaten über die Entgelteinstufung von Arbeitsplätzen und aus der Auswertung von Tarifverträgen entstandene Daten Verwendung.

\subsection{Grundüberlegung}

Der Analyse von Mikrodaten liegt ein einfaches Gedankenexperiment zugrunde, das verdeutlicht, wie sich Abwärtsnominallohnstarrheit auf die Entwicklung der beobachtbaren individuellen Entgelte auswirken sollte. Ausgangspunkt der Überlegungen ist die Auffassung, dass, wie in Abschnitt 2 geschildert, in Reaktion auf heterogene Angebots- und Nachfragestörungen auch langfristig in der Ökonomie ein ständiger Anpassungsbedarf auf Teilarbeitsmärkten herrscht. Für eine gegebene Inflationsrate in der Ökonomie und in Abwesenheit von nomineller Starrheit äußert sich dieser Anpassungsbedarf in einer Verteilung der nominellen Entgeltänderungen, die sowohl positive als auch negative Änderungen aufweist, also nicht nur Entgelterhöhungen, sondern auch Entgeltkürzungen; dies ist in Abbildung 2a beispielhaft illustriert.

\section{Abbildung 2}

Im Unterschied hierzu sollten bei Vorliegen von Abwärtsnominallohnstarrheit Entgeltkürzungen entweder gar nicht oder nur relativ selten auftreten, und stattdessen bei eigentlich vorliegendem Kürzungsbedarf durch Entgelt-,,Nullrunden“ ersetzt sein. Eine entsprechende Situation, in der die Mehrheit der gewünschten Kürzungen nicht stattfinden kann und durch konstante Entgelte ersetzt wird, ist in Abbildung 2b dargestellt. Gegenüber der Situation ohne nominelle Starrheit kommt es also zu einer charakteristischen Formveränderung der Querschnittsverteilung der Entgeltänderungen: zum einen zu einem Ausdünnen des Bereichs der 
Verteilung, der in den Bereich negativer Entgeltänderungen hineinreicht, zum anderen zu einer entsprechenden Anhäufung von Null-Änderungen. Die unmittelbare Verwertbarkeit dieser Formveränderung der Entgeltänderungsverteilung für Analysezwecke wird dadurch begrenzt, dass die Form der Ausgangsverteilung ohne Abwärtsnominallohnstarrheit unbekannt ist. Es bleibt strenggenommen unklar, ob es sich bei der beobachteten Verteilung um eine Verteilung ohne Einwirkung von nomineller Entgeltstarrheit mit einer etwas eigenwilligen Form handelt (dünner linker tail, Massepunkt bei Null), oder ob es sich um eine Verteilung handelt, die durch die Wirkung von nomineller Entgeltstarrheit zustande gekommen ist.

Um diese beiden Möglichkeiten auseinander halten zu können, sind Identifikationsstrategien vonnöten, die sicherstellen, dass tatsächliche oder vermeintliche Eigenschaften der Verteilung wie Ausdünnung und Anhäufung tatsächlich auf Abwärtsnominallohnstarrheit zurückzuführen sind.

\subsection{Identifikationsstrategien und Analyseansätze}

Die in der Literatur verwendeten Analyseansätze verwenden zwei Identifikationsstrategien, entweder einzeln oder in Kombination: Die erste besteht darin, die geschilderten Formveränderungen nutzbar zu machen, indem Annahmen über die Form der Verteilung getroffen werden, die zweite darin, auf das gemeinsame Variieren von Lage und Form der Verteilung abzustellen.

Dadurch, dass im Rahmen der ersten Identifikationsstrategie Annahmen über die Form der Verteilung getroffen werden, die ohne Abwärtsnominallohnstarrheit vorgelegen hätte, wird sichergestellt, dass beobachtete Formveränderungen der Verteilung auf nominelle Starrheit zurückzuführen sind. Der von Card und Hyslop (1997) vorgeschlagene symmetry approach basiert auf der Annahme, dass ohne nominelle Entgeltstarrheit 
Entgeltveränderungsverteilungen symmetrisch wären. Der von Borghijs (2001) angewandte normality approach geht noch einen Schritt weiter und nimmt an, dass die Entgeltänderungen ohne Rigidität normalverteilt sind. Alle beobachteten Abweichungen von diesen Annahmen können dann als Auswirkung von Abwärtsnominallohnstarrheit interpretiert werden. Der Haupteinwand gegen diese Ansätze ist, dass es in den meisten Datensätzen Hinweise darauf gibt, dass die Entgeltänderungen auch ohne Einwirkung von Entgeltstarrheit nicht symmetrisch und daher auch nicht normalverteilt sind, siehe hierzu die Kritik von McLaughlin (1999) am symmetry approach.

Die zweite Identifikationsstrategie nutzt den Umstand aus, dass die Entgeltänderungsverteilung im Zeitablauf typischerweise ihre Lage ändert. Die Lage der Entgeltänderungsverteilung spiegelt eine ganze Reihe von Einflussgrößen wieder, deren wichtigste die Inflationsrate ist: Je höher die Inflation, desto höher die mittlere Entgeltsteigerungsrate (und umgekehrt). Teil c) von Abbildung 2 stellt eine Situation ohne Einwirkung von Abwärtsnominallohnstarrheit dar, die sich von der in Teil a) dargestellten dadurch unterscheidet, dass sich die gesamte Verteilung aufgrund einer höheren Inflationsrate in der Ökonomie nach rechts verlagert hat. Sollte nun Abwärtsnominallohnstarrheit vorliegen, so bedeutet dies, dass je nach Lage der Verteilung ein unterschiedlich großer Anteil der Entgeltänderungen von Rigidität betroffen wäre. Daraus folgt, dass die Formveränderung durch Entgeltstarrheit mit den Lageveränderungen der Verteilung systematisch schwankt. Teil d) von Abbildung 2 illustriert dies. Durch die Verlagerung nach rechts liegt nur mehr ein kleinerer Teil der Verteilung im negativen Bereich und wird von der Wirkung der Abwärtsnominallohnstarrheit erfasst. Entsprechend fällt auch die Anhäufung bei Null geringer aus. Die zweite Strategie stellt genau auf dieses gemeinsame Variieren von Lage und Form der Entgeltänderungsverteilung ab und akzeptiert Eigenschaften wie Ausdünnung und Anhäufung nur dann als Anzeichen von nomineller Entgeltrigidität, wenn sie systematisch mit der Lage der Verteilung schwanken. 
Mehrere Analyseansätze basieren auf dieser zweiten Identifikationsstrategie, nämlich der skewness-location approach, der histogram-location approach und der earnings-function approach.

Der auf McLaughlin (1994) zurückgehende skewness-location approach erfasst die Formveränderungen der Entgeltänderungsverteilung durch Schiefemaße wie den Schiefe-Koeffizienten und überprüft durch Regressionsanalysen, ob tatsächlich ein gemeinsames Variieren von Lage und Form der Verteilung vorliegt. Ein schwerwiegender Nachteil dieses Ansatzes liegt darin, dass zwar die Existenz von Abwärtsnominallohnstarrheit geprüft, ihr Ausmaß aber nicht bewertet werden kann. Quantitative Aussagen sind dagegen mit Hilfe der folgenden beiden Ansätze möglich. Der von Kahn (1997) vorgeschlagene histogram-location approach bildet die beobachteten Entgeltänderungsverteilungen durch Histogramme ab und prüft, ob sich die Formveränderungen der Histogramme im Zeitablauf durch ein systematisches Zusammenspiel von nomineller Entgeltstarrheit und Lageveränderungen erklären lassen. In den beiden bisher genannten Ansätzen, die das zweite Identifikationsprinzip nutzen, wird unterstellt, dass die Form der ungehinderten Entgeltänderungsverteilung im Zeitablauf konstant bleibt und dass von Messproblemen abstrahiert werden kann. Beide Annahmen sind kritisiert worden. Die Form der Verteilung kann sich im Zeitablauf beispielsweise dadurch ändern, dass sich die Zusammensetzung der Arbeitnehmerschaft ändert. Messfehler können unter anderem dadurch entstehen, dass sich die Befragten nicht exakt an die wahren Entgeltwerte erinnern oder diese nicht korrekt berichten. Streuen die berichteten Werte zufällig um die wahren Werte, so hat dies ohne weitere Vorkehrungen eine Unterschätzung des wahren Rigiditätsgrades zur Folge, siehe Knoppik und Beissinger (2003). Treten dagegen Rundungsfehler auf, so führt dies $\mathrm{zu}$ einer $\mathrm{zu}$ hohen beobachteten Zahl von konstanten Entgeltwerten und einer möglichen Überschätzung, siehe hierzu Smith (2000). 
Der von Altonji und Devereux (2000) eingeführte earnings-function approach berücksichtigt die Kritik an beiden Annahmen, indem die Entgeltänderungsverteilung durch individuelle, sektorale und aggregierte Variablen erklärt wird. Im zugehörigen Schätzansatz wird geprüft, ob die Modellierung von Abwärtsnominallohnstarrheit die Erklärung der beobachteten Formveränderungen der Entgeltänderungsverteilung verbessert. Hierbei finden genau genommen allerdings im Rahmen der strukturellen Modellierung der Lohnbildung beide Identifizierungsstrategien Verwendung. Das Auftreten von Messfehlern wird durch eine explizite Modellierung bei der Schätzung ebenfalls berücksichtigt.

Deskriptive Analysen von Entgeltänderungsverteilungen im Hinblick auf Abwärtsnominallohnstarrheit seien an dieser Stelle ebenfalls erwähnt, da eine große Anzahl von Studien sich auf diese Art der Auswertung der Daten beschränkt. Bewertungen hinsichtlich der Existenz und des Ausmaßes von nomineller Entgeltstarrheit werden in diesem Fall typischerweise anhand einer undeklarierten Mischung aus beiden Identifikationsstrategien vorgenommen, in deren Rahmen sowohl vermeintliche Abweichungen von der Form der Verteilung ohne Rigiditätseinwirkung als auch Veränderungen beispielsweise der Anhäufung bei Null eine nicht exakt charakterisierte Rolle spielen.

Abschließend bleibt festzuhalten, dass sich die angesprochenen Analyseansätze vor allem darin unterscheiden, ob sie quantitative Aussagen über das Ausmaß der Abwärtsnominallohnstarrheit ermöglichen und ob sie in der Lage sind, in den Daten enthaltene Messfehler zu berücksichtigen. ${ }^{6}$

6 Weitere Eigenschaften und Annahmen sind eher technischer Natur und werden hier nicht weiter diskutiert, siehe hierzu Beissinger und Knoppik (2001), Knoppik (2001) und Stiglbauer (2002). 


\subsection{Ergebnisse: Existenz und Ausmaß von Abwärtsnominallohnstarrheit}

Tabelle 2 listet ausgewählte Studien zur Abwärtsnominallohnstarrheit in Mikrodaten mit grundlegenden Informationen über die verwendeten Daten und Analyseansätze sowie die gefundenen Ergebnisse auf. Die Beschränkung auf eine Auswahl erfolgt zum einen aus Platzgründen, zum anderen aufgrund der gerade skizzierten Kritik an der rein deskriptiven Vorgehensweise sowie am symmetry approach und am skewness-location approach. ${ }^{7}$ Auf den ersten Blick fällt auf, dass Einschätzungen des Ausmaßes der Abwärtsnominallohnstarrheit praktisch das gesamte Spektrum möglicher Ergebnisse abdecken. Damit drängt sich die Frage auf, ob irgendeine Gesetzmäßigkeit hinter dieser starken Variation steckt, und, wenn ja, welche.

\section{Tabelle 2}

Eine erste Vermutung folgt direkt aus der Hypothese, die institutionellen Gegebenheiten bestimmten das Ausmaß der Rigidität. Die Aufschlüsselung der Ergebnisse aus Tabelle 2 nach Arbeitsmarktregimes und Rigiditätsgrad lässt allerdings kein derartiges Muster erkennen, wie aus Tabelle $3 \mathrm{zu}$ entnehmen ist. Sowohl für angelsächsische, als auch für kontinentaleuropäische Länder wird jeweils das gesamte Ergebnisspektrum von niedrig bis sehr hoch abgedeckt.

\section{Tabelle 3}

Erfolgreicher ist die Gegenüberstellung von ermitteltem Rigiditätsgrad und der Art der Messfehlerbehandlung. Tabelle 4 zeigt, dass Studien von messfehlerfreien Daten oder unter Verwendung von expliziten Messfehlermodellen durchweg hohe oder sehr hohe Rigiditäts-

7 Die wichtigsten Studien, die diese drei Ansätze verwenden, werden im folgenden nach Ansätzen und Ländern gruppiert aufgeführt. Deskriptive Analysen: Borghijs (2001) für Belgien; Nickell und Quintini (2001), Smith (2000, 2002) für Großbritannien; Dessy (2002) für die EU12-Staaten; Gottschalk (2002), Wilson (1999) für die USA. Symmetry approach: Charlton (2000) für Australien. Skewness-location approach: Dwyer und Leong (2000) für Australien; Christofides und Stengos (2002, 2003) für Kanada; Chapple (1996) für Neuseeland; Christofides und Stengos (2001), Lebow, Stockton und Washer (1995), McLaughlin (1999) für die USA. 
grade finden, während Studien ohne Messfehlerkontrolle durchweg nur mittlere oder niedrige Rigiditätsgrade ermitteln.

\section{Tabelle 4}

Diese Beobachtungen legen den Schluss nahe, dass elaborierte Verfahren mit Berücksichtigung der Messfehlerproblematik durchweg ein hohes Ausmaß an Rigidität finden, unabhängig von den vorliegenden Arbeitsmarktinstitutionen. ${ }^{8}$ Allerdings sind die Messfehler in den Entgeltvariablen der meist verwendeten Haushaltssurveypanels und geeignete Korrekturverfahren derzeit noch Gegenstand der wissenschaftlichen Diskussion, so dass diese Ergebnisse als vorläufig angesehen werden müssen, vgl. beispielsweise Gottschalk (2002).

Im Gegensatz zum Ausmaß der Abwärtsnominallohnstarrheit werden deren Gründe in nur zwei Studien auf der Basis von Mikrodaten überhaupt thematisiert. Dessy (2002) untersucht die Rolle von Arbeitsmarktinstitutionen für den Rigiditätsgrad und Smith (2002) prüft die mögliche Rolle des Fairness-Arguments für die Vermeidung von Lohnkürzungen. In beiden Fällen wird der Rigiditätsgrad allerdings nur durch den deskriptiven Ansatze erfasst. Wegen der bereits geschilderten Probleme dieser Vorgehensweise sind Vorbehalte gegenüber den Ergebnissen beiden Studien anzumelden.

\section{Quantitative Abschätzung der makroökonomischen Konsequenzen}

In diesem Abschnitt soll aufgezeigt werden, welche realen makroökonomischen Konsequenzen aus der mikroökonometrischen Evidenz folgen. ${ }^{9}$ Hierzu ist auf die Überlegungen aus

8 Für Großbritannien existieren derzeit nur Studien auf Basis von Mikrodaten, die diese Verfahren nicht verwenden und zu abweichenden Ergebnissen gelangen. So finden Smith (2000) und Nickell und Quintini (2001) keine bzw. sehr geringe Nominallohnstarrheit nach unten.

9 Dies schließt neuere Studien allein auf Basis von Makroevidenz aus, wie z. B. Wyplosz (2001) und Dickens (2001). 
Abschnitt 2 zurückzugreifen. Deren Ergebnis war, dass bei unvollkommenem Wettbewerb, heterogenen Angebots- und Nachfragestörungen und Abwärtsnominallohnstarrheit die Höhe der Langfrist-Gleichgewichtsarbeitslosigkeit für niedrige Inflationsraten von der Höhe der Inflationsrate abhängt.

Aufgrund der präsentierten Evidenz können die Existenz und ein nicht unerhebliches Ausmaß von nomineller Lohnstarrheit in vielen Ländern als nachgewiesen gelten. Zur Abschätzung der realen Wirkungen dieser Lohnstarrheit ist es allerdings nicht ausreichend, nur $\mathrm{zu}$ wissen, welcher Anteil der gewünschten Kürzungen aufgrund von Abwärtsnominallohnstarrheit unterbleibt. Vielmehr ist ausschlaggebend, wie viele Entgeltkürzungen überhaupt gewünscht werden. Wie bereits dargestellt, hängt dies wesentlich von der Inflationsrate ab und kann zumindest im earnings-function approach auf Basis der Schätzergebnisse berechnet werden. ${ }^{10}$ Neben dem Anteil der verhinderten Entgeltänderungen an der Gesamtzahl aller Lohnänderungen kann auch berechnet werden, wie stark die Erhöhung der durchschnittlichen realen Entgeltänderung durch Abwärtsnominallohnstarrheit ausfällt und welches Ausmaß die Erhöhung des durchschnittlichen realen Entgeltniveaus durch Abwärtsnominallohnstarrheit aufweist. Beide Erhöhungen hängen von der Höhe der Inflationsrate ab. Die durch die Nominallohnstarrheit verursachte Anhebung des Reallohnniveaus kann ihrerseits verwendet werden um abzuschätzen, wie stark die gleichgewichtige Arbeitslosenquote $u^{*}$ gegenüber einer Situation ohne Lohnstarrheit ansteigen muss, um inflationsstabil zu sein. Da die Erhöhung des durchschnittlichen realen Entgeltniveaus von der Inflation abhängt, gilt dies auch für die gleichgewichtige Arbeitslo-

10 Diese Berechnung ist nur möglich und sinnvoll, wenn durch den Analyseansatz zum einen die gewünschte Entgeltänderungsverteilung quantitativ bestimmt wird und zum anderen nicht durch unberücksichtigte Messfehler verbreitert ist. Beide Voraussetzungen zusammen werden derzeit nur vom earnings-function approach erfüllt. Die Verletzung der zweiten Voraussetzung ist vermutlich für das von Lebow, Saks und Wilson (1999) gefundene und von Castellanos (2001) diskutierte micro-macro puzzle verantwortlich. Dieses besteht darin, dass die Mikroevidenz in diesen Studien wegen breiter Entgeltänderungsverteilungen überaus starke Auswirkungen der Abwärtsnominallohnstarrheit suggeriert, die in Makrodaten dann nicht nachgewiesen werden können. 
senquote $u^{*}$, d. h. ceteris paribus hängt das Ausmaß der Zusatz-Arbeitslosigkeit von der Inflationsrate $a b$.

Zwei Studien, Knoppik und Beissinger (2003) und Devicienti (2003), quantifizieren für Deutschland und Italien die Zusatz-Arbeitslosigkeit. Die graphische Darstellung der entsprechenden Ergebnisse findet sich in Abbildung 3 in Form von normierten LangfristPhillipskurven; diese geben auf der Abszisse angetragen die Höhe der Zusatz-Arbeitslosigkeit in Abhängigkeit von der Inflationsrate an. In beiden Fällen sind die nachteiligen Auswirkungen von Null-Inflation auf die gleichgewichtige Arbeitslosenquote etwas geringer als die von Akerlof, Dickens und Perry (1996) für die USA gefundenen und betragen etwa ein bis zwei Prozentpunkte. ${ }^{11}$

\section{Abbildung 3}

Schlussfolgerungen über das Verhalten der Ökonomie bei Niedriginflation, wie die gerade gezogenen, werden häufig im Stil der sogenannten Lucas-Kritik in Frage gestellt: Würde es nicht angesichts anhaltender Niedriginflation zu Verhaltensänderungen kommen, die das Phänomen der Geldillusion und damit auch die Abwärtsnominallohnstarrheit verschwinden lassen? Gegen diese Kritik ist einzuwenden, dass ein Teil der mikroökonometrischen Evidenz und der Evidenz aus Befragungsstudien bereits auf Niedriginflationsphasen beruht (Deutschland, Schweden und Schweiz). Zudem legt der psychologische Erklärungsansatz für Nominallohnstarrheit nahe, dass niedrige Inflation die Verwendung des nominellen Frames sogar verstärkt. Beispielsweise liegt Evidenz dafür vor, dass die bei höherer Inflation abgelegte Geldillusion bei niedrigerer Inflation wieder zurückkehrt, siehe hierzu Shafir, Diamond und Tversky (1997).

11 Auch andere Studien auf Basis des earnings-function approach, Altonji und Devereux (2000), Fehr und Goette (2000), und Fehr, Goette und Pfeiffer (2002), untersuchen reale Auswirkungen der Abwärtsnominallohnstarrheit. In diesen Arbeiten stehen jedoch die Wirkungen auf andere reale Größen im Vordergrund, die Auswirkungen auf die aggregierte gleichgewichtige Beschäftigung sind daher nicht unmittelbar ersichtlich. 


\section{Zusammenfassung und wirtschaftspolitische Implikationen}

In diesem Beitrag werden die wichtigsten Erkenntnisse aus Befragungsstudien, experimentellen Untersuchungen und vor allem mikroökonometrischen Studien zu Existenz, Ausmaß und realen Konsequenzen von Abwärtsnominallohnstarrheit zusammengefasst, erklärt und bewertet. Nach unserer Auffassung verdichtet sich bereits jetzt die anwachsende Evidenz zu folgendem vorläufigen Gesamtbild: Erstens, die Existenz von Abwärtsnominallohnstarrheit scheint durch die empirische Evidenz bestätigt. Zweitens, unter Berücksichtigung der Messfehlerproblematik kommt man zum Schluss, dass das Ausmaß der Entgeltstarrheit erheblich ist. Drittens, die realen Auswirkungen von nomineller Entgeltstarrheit sind zwar möglicherweise etwas geringer als bei Akerlof, Dickens und Perry (1996), sie liegen aber in einer keinesfalls zu vernachlässigenden Größenordnung.

Für die Wirtschaftspolitik ergeben sich grundsätzlich drei Möglichkeiten für den Umgang mit der gefundenen Abwärtsnominallohnstarrheit. Entweder man baut darauf, dass das Problem verschwindet, indem sich die ökonomischen Akteure an das Niedriginflationsumfeld anpassen, oder man setzt an den Ursachen der nominellen Starrheit an und versucht ihr Ausmaß zu verringern, oder man passt die Geldpolitik an die vorliegende nominelle Entgeltstarrheit an.

Sowohl die präsentierte psychologische Evidenz als auch die Tatsache, dass ein erheblicher Teil der mikroökonometrischen Evidenz aus ausgesprochenen Niedriginflationsphasen stammt, lassen allerdings nicht erwarten, dass sich das Phänomen der Abwärtsnominallohnstarrheit bei Niedrig-Inflation durch Abwarten in Wohlgefallen auflöst.

Nach dem institutionellen Ansatz zur Erklärung der Abwärtsnominallohnstarrheit würde sich durch geeignetes Verändern der Arbeitsmarktinstitutionen, entweder gezielt im Hinblick auf Reduktion nomineller Entgeltstarrheit oder im Rahmen allgemeiner struktureller Reformen mit anderen primären Zielsetzungen, das Ausmaß der Abwärtsnominallohnstarrheit 
verringern lassen. Die Evidenz aus angelsächsischen Ländern deutet allerdings darauf hin, dass es auch ohne die entsprechenden unterstützenden institutionellen Regelungen zur Realisierung des psychologisch bedingten Phänomens der Abwärtsnominallohnstarrheit kommt. Es ist daher zu vermuten, dass sich institutionelle Reformen in dieser Hinsicht als weitgehend wirkungslos erweisen werden.

Damit ist die Wirtschaftspolitik auf die dritte Alternative angewiesen, nämlich die Anpassung der Geldpolitik an die Abwärtsnominallohnstarrheit. Dies schlägt sich vor allem bei der Bestimmung der Höhe des Inflationsziels nieder. Hierbei sind neben den Kosten sehr niedriger Inflationsraten natürlich auch deren Vorteile in Ansatz zu bringen, so dass sich aus der Evidenz zur Abwärtsnominallohnstarrheit allein nicht unmittelbar Aussagen über die optimale Inflationsrate ableiten lassen. ${ }^{12}$ Allerdings steht die EZB wegen ihres im internationalen Vergleich besonders niedrigen Inflationsziels auch nach der jüngsten Überprüfung ihrer geldpolitischen Strategie unter einem gewissen Rechtfertigungsdruck.

12 Vgl. hierzu z. B. Edey (1994), Briault (1995) und Thornton (1996). 


\section{Literatur}

Agell, Jonas und Bennmarker, Helge (2002), 'Wage policy and endogenous wage rigidity: a representative view', CESifo working paper series 751, July.

Agell, Jonas und Lundborg, Per (2003), 'Survey Evidence on Wage Rigidity: Sweden in the 1990s', in: Scandinavian Journal of Economics, 105(1), 15-29.

Akerlof, George A.; Dickens, William T. und Perry, George L. (1996), 'The Macroeconomics of Low Inflation [einschließlich Kommentare von Gordon und Mankiw]', in: Brookings Papers on Economic Activity, (1), 1-59 [60-76].

Altonji, Joseph G. und Devereux, Paul J. (2000), 'Is there Nominal Wage Rigidity? Evidence from Panel Data', in: Research in Labor Economics, 19, 383-431.

Beissinger, Thomas und Knoppik, Christoph (2001), 'Downward Nominal Rigidity in West-German Earnings 1975-1995', in: German Economic Review, 2(4), 385-418.

Bewley, Truman F. (1999), Why Wages Don't Fall During a Recession, Cambridge, MA, Cambridge University Press.

Blinder, Alan S. und Choi, Don H. (1990), 'A Shred of Evidence of Theories of Wage Stickiness', in: Quarterly Journal of Economics, 105(3), 1003-1015.

Borghijs, Alain (2001), 'Are nominal wages downwardly rigid? Evidence from Belgian microdata', mimeo, Antwerp.

Briault, Clive (1995), 'The Costs of Inflation', in: Bank of England Quarterly Bulletin, February, 3345.

Campbell, Carl M. und Kamlani, Kulan S. (1997), 'The Reasons for Wage Rigidity: Evidence from a Survey of Firms', in: Quarterly Journal of Economics, 112(3), 759-89.

Card, David und Hyslop, Dean (1997), 'Does Inflation "Grease the Wheels of the Labor Market"? [einschließlich Kommentar von Shea]', in: Romer, Christina D. und Romer, David H. (Hrsg.), Reducing Inflation - Motivation and Strategy, Chicago, London, University of Chicago Press, 71114 [114-122].

Castellanos, Sara G. (2001), 'Downward Nominal Wage Rigidities and Employment: Microeconomic Evidence of Mexico', mimeo, Bank of Mexico, January.

Chapple, Simon (1996), 'Money wage rigidity in New Zealand', in: Labour Market Bulletin [Department of Labour], 1996(2), 23-50.

Charlton, Andrew (2000), 'Nominal Wage Rigidity in the Australian Labour Market', mimeo, Reserve Bank of Australia.

Christofides, Louis N. und Leung, Man Tuen (2003), Nominal Wage Rigidity in Contract Data: A Parametric Approach, in: Economica, erscheint demnächst.

Christofides, Louis N. und Stengos, Thanasis (2001), 'A non-parametric Test of the Symmetry of the PSID Wage-Change Distribution', in: Economics Letters, 71(3), June, 363-368.

Christofides, Louis N. und Stengos, Thanasis (2002), 'The Symmetry of the Wage-Change Distribution: Survey and Contract Data', in: Empirical Economics, 27(4), 705-723.

Christofides, Louis N. und Stengos, Thanasis (2003), 'Wage Rigidity in Canadian Collective Bargaining Agreements', in: Industrial and labor relations review, 56(3), 429-448.

Decressin, Anja und Decressin, Jörg (2002), 'On Sand and the Role of Grease in Labor Markets: How Does Germany Compare?', IMF Working Paper 02/164, September.

Dessy, Orietta (2002), 'Nominal wage rigidity and institutions: micro-evidence from the Europanel', mimeo, CREST, July 10th.

Devicienti, Franceso (2003), 'Downward Nominal Wage Rigidity in Italy: Evidence and Consequences', mimeo, LABORatorio Riccardo Revelli, January. 
Dickens, William T. (2001), 'Comment [zu Wyplosz]', in: Europäische Zentralbank (Hrsg.), Why price stability?, Frankfurt, 34-45.

Dwyer, Jacqeline und Leong, Kenneth (2000), 'Nominal wage rigidity in Australia', Research Discussion Paper [Reserve Bank of Australia] 2000-08, November.

Economist (2000a), 'How low can you go', in: Economist, 11.11.2000, 126.

Economist (2000b), 'Why wages do not fall in recessions', in: Economist, 26.02.2000, 104.

Economist (2002), 'Wobbly Pillars', in: Economist, 21.12.2002, 107.

Edey, Malcolm (1994), 'Costs and Benefits of Moving from Low Inflation to Price Stability', in: OECD Economic Studies, 23, 109-30.

Europäische Zentralbank (Hrsg.) (2001), Why price stability?, Frankfurt.

Europäische Zentralbank (2003), Overview of the background studies for the reflections on the ECB's monetary policy strategy, Frankfurt.

Fehr, Ernst und Falk, Armin (1999), 'Wage Rigidity in a Competitive Incomplete Contract Market', in: Journal of Political Economy, 107(1), 106-34.

Fehr, Ernst und Gächter, Simon (2000), 'Fairness and Retaliation: The Economics of Reciprocity', in: Journal of Economic Perspectives, 14(3), 159-81.

Fehr, Ernst und Goette, Lorenz (2000), 'Robustness and Real Consequences of Nominal Wage Rigidity', IERE Working Paper Series 44, May.

Fehr, Ernst und Tyran, Jean-Robert (2001), 'Does Money Illusion Matter?', in: American Economic Review, 91(5), 1239-1262.

Fehr, Ernst; Goette, Lorenz und Pfeiffer, Friedhelm (2002), 'Dimensions and Consequences of Wage Rigidities in Germany', mimeo, ZEW - Zentrum für europäische Wirtschaftsforschung, February.

Franz, Wolfgang und Pfeiffer, Friedhelm (2003), 'Zur ökonomischen Rationalität von Lohnrigiditäten aus der Sicht von Unternehmen', in: Jahrbücher für Nationalökonomie und Statistik (Journal of Economics and Statistics), 223(1), 23-57.

Friedman, Milton (1968), 'The Role of Monetary Policy', in: American Economic Review, 58(1), 1-17.

Gottschalk, Peter (2002), 'Downward nominal wage flexibility - real or measurement error?', mimeo, Boston College, May.

Holden, Steinar (2002), 'The Costs of Price Stability - Downward Nominal Wage Rigdity in Europe', NBER Working paper series 8865, March.

IMF (1999), 'Safeguarding Macroeconomic Stability at Low Inflation', in: IMF, World Economic Outlook, 92-126.

Kahn, Shulamit (1997), 'Evidence of Nominal Wage Stickiness from Microdata', in: American Economic Review, 87(5), 993-1008.

Kaufman, R. (1984), 'On wage stickiness in Britains's competitive sector', in: British Journal of Industrial Relations, 22, 101-112.

Knoppik, Christoph (2001), 'Nominal wage rigidity', mimeo, Regensburg, October.

Knoppik, Christoph und Beissinger, Thomas (2002), 'Measuring the Extent of Downward Nominal Wage Rigidity in Variants of the Earnings-Function Approach', mimeo, Regensburg, July.

Knoppik, Christoph und Beissinger, Thomas (2003), 'How Rigid are Nominal Wages? Evidence and Implications for Germany', in: Scandinavian Journal of Economics, 105(4).

Knoppik, Christoph und Dittmar, Jens (2002), 'A Semi-Parametric Analysis of Downward Nominal Wage Rigidity in the GSOEP 1984-2000', University of Regensburg Discussion Papers in Economics 374, September.

Landmann, Oliver und Jerger, Jürgen (1999), Beschäftigungstheorie, Berlin u.a., Springer.

Layard, Richard; Nickell, Stephen und Jackman, Richard (1991), Unemployment - Macroeconomic Performance and the Labour Market, Oxford, Oxford University Press. 
Lebow, David E.; Saks, Raven E. und Wilson, Beth Anne (1999), 'Downward Nominal Wage Rigidity: Evidence from the Employment Cost Index', Finance and Economics Discussion Series 99/31, July.

Lebow, David E.; Stockton, David und Washer, William (1995), 'Inflation, Nominal Wage Rigidity, and the Efficiency of Labor Markets', Finance and Economics Discussion Series 95-45, October.

McLaughlin, Kenneth J. (1994), 'Rigid Wages?', in: Journal of Monetary Economics, 34, 383-414.

McLaughlin, Kenneth J. (1999), 'Are Nominal Wage Changes Skewed Away From Wage Cuts? [einschließlich Kommentar von Startz]', in: Federal Reserve Bank of St. Louis Review, 81(3), 117132 [133-136].

Nickell, Stephen und Quintini, Glenda (2001), 'Nominal Wage Rigidity and the Rate of Inflation', Center for economic performance discussion paper series 0489, March.

OECD (2002), 'Inflation persistence in the euro area', in: OECD Economic Outlook, 72, 352-365.

Phelps, Edmund S. (1967), 'Phillips Curves, Expectations of Inflation, and Optimal Unemployment over Time', in: Economica, 34(135), 254-281.

Rees, Albert (1973), 'The Economics of Work and Pay', New York, Harper and Row.

Shafir, Eldar; Diamond, Peter A. und Tversky, Amos (1997), 'Money Illusion', in: Quarterly Journal of Economics, 112(2), 341-374.

Smith, Jennifer C. (2000), 'Nominal Wage Rigidity in the United Kingdom', in: Economic Journal, 110, C176-C195.

Smith, Jennifer C. (2002), 'Pay cuts and morale: a test of downward nominal rigidity', Warwick economic research papers 649, September.

Stiglbauer, Alfred (2002), 'Identification of Wage Rigidities in Microdata - a Critical Literature Review', in: Focus on Austria, 2002(3), 110-126.

Thornton, D. L. (1996), 'The Costs and Benefits of Price Stability: An Assessment of Howitt's Rule', in: Federal Reserve Bank of St. Louis Review, 78(2), March.

Tobin, James (1972), 'Inflation and Unemployment', in: American Economic Review, 62(1), 1-18.

Wilson, Beth Anne (1999), 'Wage Rigidity: A Look Inside the Firm', Finance and Economics Discussion Series 99/22, April.

Wyplosz, Charles (2001), 'Do We Know How Low Should Inflation Be?', in: European Central Bank (Hrsg.), Why price stability?, Frankfurt, 15-33. 


\section{Abbildungen}

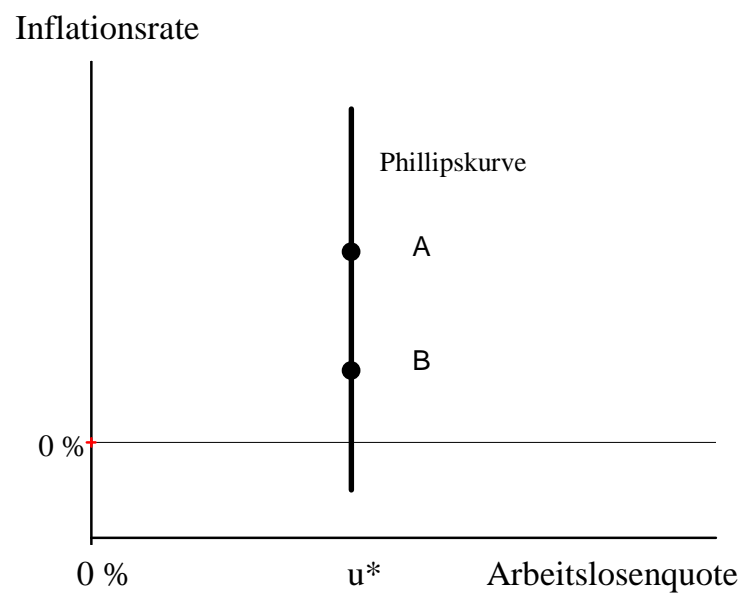

a) Ohne Abwärtsnominallohnstarrheit

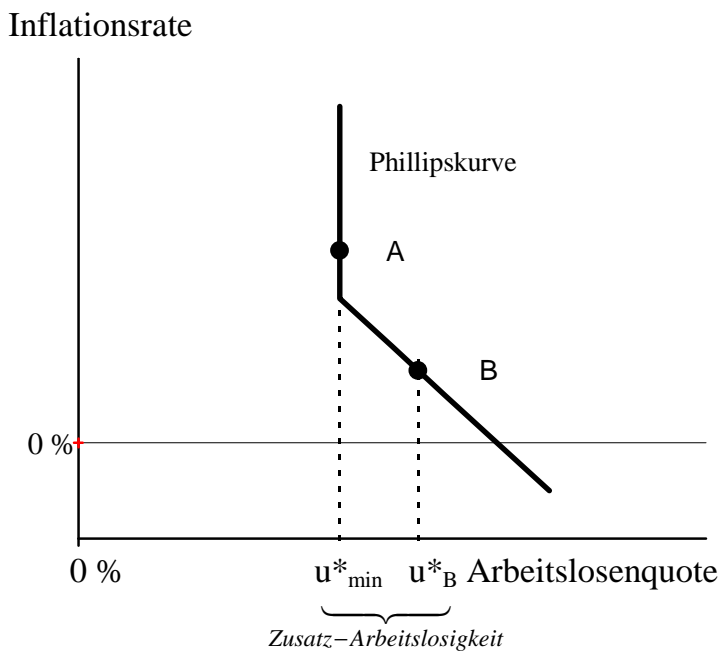

b) Mit Abwärtsnominallohnstarrheit

Abbildung 1 Langfristige Phillipskurven bei flexbiblen und nach unten starren Nominallöhnen 


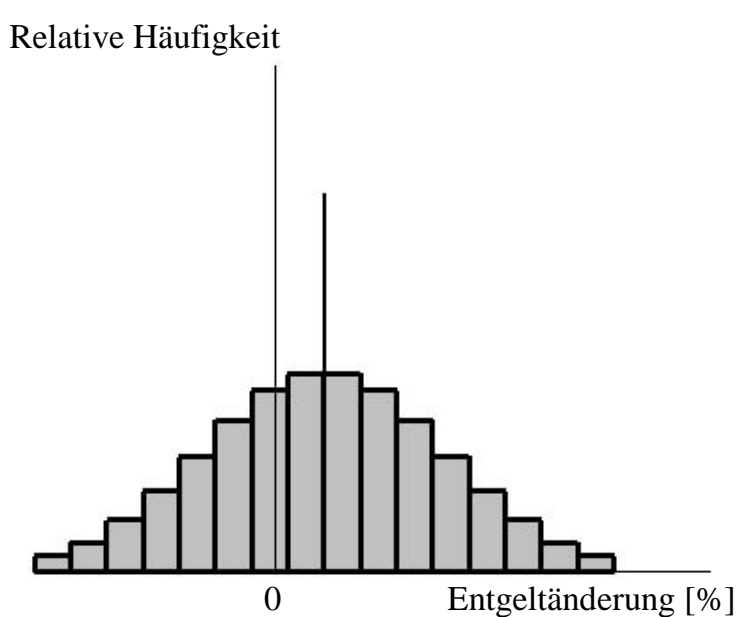

a) Ohne Abwärtsnominallohnstarrheit - niedrige Inflation

Relative Häufigkeit

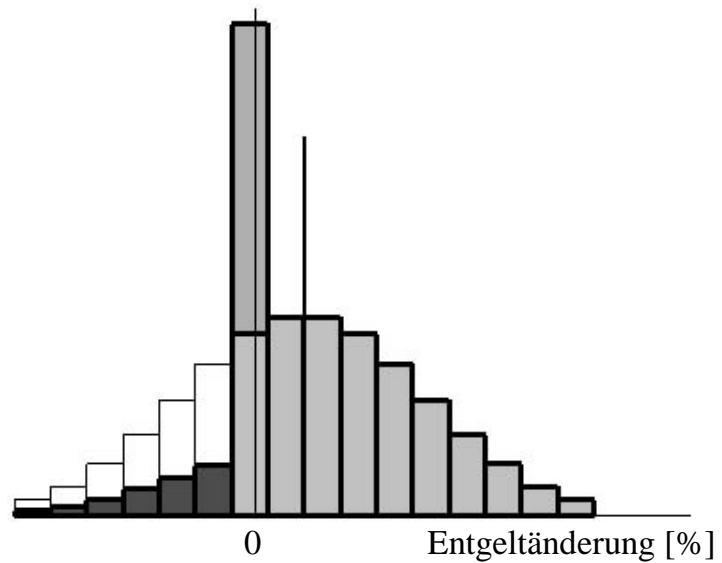

b) Mit Abwärtsnominallohnstarrheit - niedrige Inflation

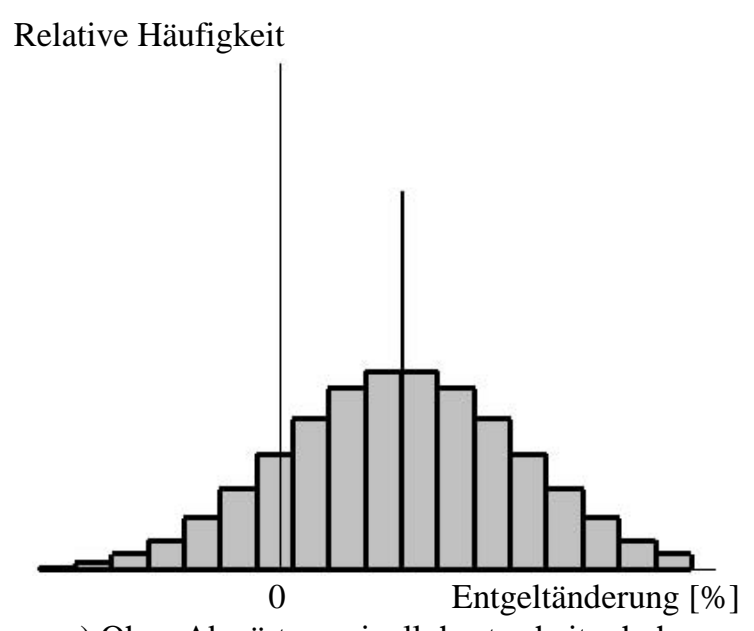

c) Ohne Abwärtsnominallohnstarrheit - hohe Inflation

Relative Häufigkeit

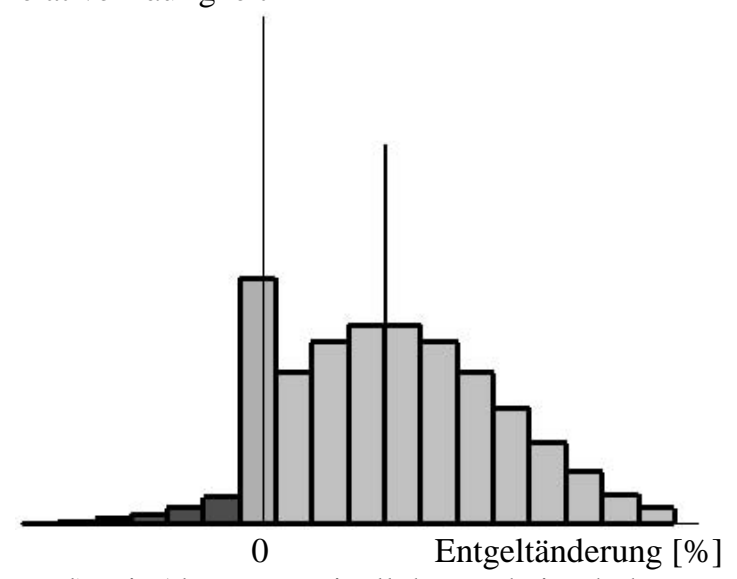

d) Mit Abwärtsnominallohnstarrheit - hohe Inflation

Abbildung 2 Entgeltänderungsverteilungen mit und ohne Abwärtsnominallohnstarrheit Anmerkung: Die kurzen senkrechten Linien markieren die Lage der Entgeltänderungsverteilungen. 


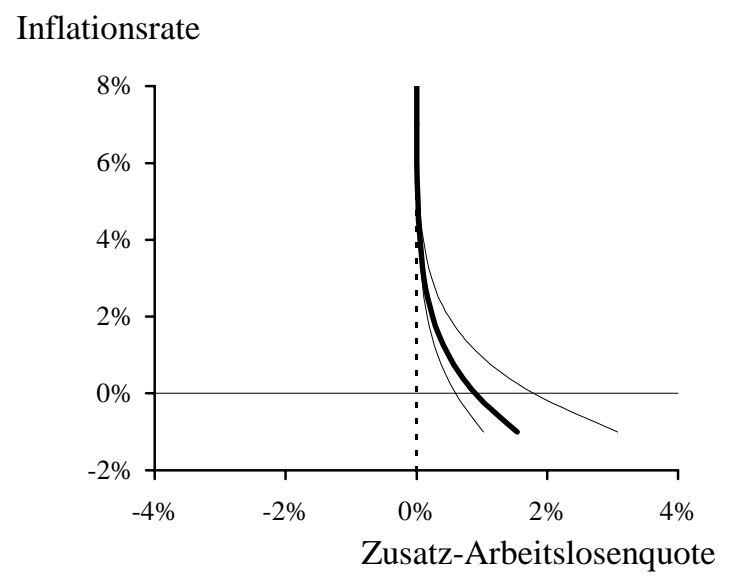

a) Deutschland
Inflationsrate

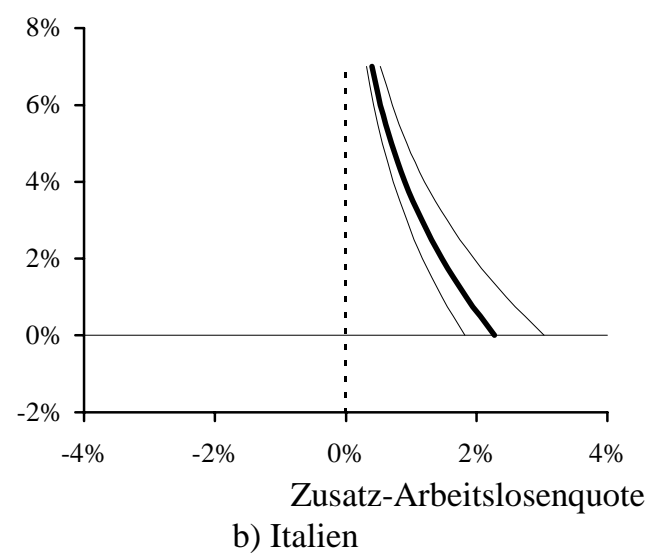

Abbildung 3 Normierte langfristige Phillipskurven

Anmerkung: Die Langfrist-Phillipskurven sind jeweils für alternative Werte des Parameters $a$ angegeben, der die Reaktion der Inflation auf die Abweichungen der tatsächlichen Arbeitslosigkeit von der NAIRU erfasst: $a=.2, .4, .6$ (Deutschland) bzw. $a=.3, .4, .5$ (Italien).

Quellen: Deutschland, Knoppik und Beissinger (2003), fig. 2; Italien, Devicienti (2003), tab. 9, p. 53. 


\section{Tabellen}

Tabelle 1: Befragungsstudien

\begin{tabular}{|c|c|c|c|c|c|}
\hline & & & Ausmaß & \multicolumn{2}{|c|}{ Ursachen } \\
\hline Land & Studie & Daten & Rigidität & Fairness & Institutionen \\
\hline 1 & 2 & 3 & 4 & 5 & 6 \\
\hline DEU & $\begin{array}{l}\text { Franz und } \\
\text { Pfeiffer (2003) }\end{array}$ & $\begin{array}{l}801 \text { Unternehmen mit mehr als } 10 \\
\text { Beschäftigten aus } 6 \text { Wirtschaftsbereichen } \\
\text { des Verarbeitenden Gewerbes und } \\
\text { Dienstleistungssektors im Jahr 2000. }\end{array}$ & $\begin{array}{l}\text { Keine } \\
\text { Angabe }\end{array}$ & $\mathrm{X}$ & $\mathrm{X}$ \\
\hline GBR & Kaufman (1984) & $\begin{array}{l}20 \text { kleine Firmen ohne und } 6 \text { große Firmen } \\
\text { mit Gewerkschaftseinfluss aus } \\
\text { Verarbeitendem Gewerbe und } \\
\text { Dienstleistungssektor im Jahr } 1982\end{array}$ & $\begin{array}{l}\text { Sehr } \\
\text { hoch }\end{array}$ & $X$ & -- \\
\hline SWE & $\begin{array}{l}\text { Agell und } \\
\text { Lundborg } \\
(2003)\end{array}$ & $\begin{array}{l}\text { Panel aus } 157 \text {, überwiegend großen, } \\
\text { Firmen aus dem Verarbeitenden Gewerbe } \\
\text { für } 1991 \text { und } 1998\end{array}$ & $\begin{array}{l}\text { Sehr } \\
\text { hoch }\end{array}$ & $X$ & $\mathrm{X}$ \\
\hline & $\begin{array}{l}\text { Agell und } \\
\text { Bennmarker } \\
(2002)\end{array}$ & $\begin{array}{l}885 \text { Firmen aus dem Verarbeitendem } \\
\text { Gewerbe, Dienstleistungs- u. öffentlichem } \\
\text { Sektor im Jahr 1999. Darunter } 300 \text { kleine } \\
\text { Firmen. }\end{array}$ & $\begin{array}{l}\text { Sehr } \\
\text { hoch }\end{array}$ & $X$ & $X$ \\
\hline USA & Bewley (1999) & $\begin{array}{l}335 \text { Manager, Gewerkschaftsführer, } \\
\text { Arbeitsvermittler, etc. von 1992-1994 }\end{array}$ & $\begin{array}{l}\text { Sehr } \\
\text { hoch }\end{array}$ & $X$ & -- \\
\hline & $\begin{array}{l}\text { Blinder und } \\
\text { Choi (1990) }\end{array}$ & $\begin{array}{l}13 \text { große Firmen aus Verarbeitendem } \\
\text { Gewerbe und } 6 \text { große Firmen aus } \\
\text { Dienstleistungssektor in } 1988\end{array}$ & Mittel & $X$ & -- \\
\hline & $\begin{array}{l}\text { Campbell und } \\
\text { Kamlani (1997) }\end{array}$ & $\begin{array}{l}111 \text { große Firmen und } 73 \text { kleinere Firmen } \\
\text { aus Verarbeitendem Gewerbe und } \\
\text { Dienstleistungssektor in 1993/94 }\end{array}$ & $\begin{array}{l}\text { Keine } \\
\text { Angabe }\end{array}$ & $X$ & -- \\
\hline
\end{tabular}

Anmerkungen: Die Studien sind gemäß den folgenden Sortierkriterien angeordnet: 1. Ländergruppen (EU, Europa, Rest der Welt); 2. Länder, 3. Autoren.

Das angegebene Ausmaß der Nominallohnrigidität bezieht sich auf den Anteil der Firmen, die in der Vergangenheit keine Nominallohnkürzungen vorgenommen haben. Dieser Anteil hängt wie im Text erläutert von der Höhe der Inflationsrate und der Rate des Produktivitätswachstums ab und ist als quantitatives Maß für Rigidität daher mit Vorsicht zu interpretieren. 
Tabelle 2: Ausgewählte Mikrodaten-Studien und ihre Ergebnisse

\begin{tabular}{|c|c|c|c|c|c|}
\hline Land & Studie & Daten $^{(a)}$ & Ansatz & $\begin{array}{l}\text { Messfehler } \\
\text { Kontrolle }^{(b)}\end{array}$ & $\begin{array}{l}\text { Ausmaß der } \\
\text { Rigidität }^{(c)}\end{array}$ \\
\hline \multirow[t]{5}{*}{ DEU } & Beissinger und Knoppik (2001) & IABS & HLA & nein & niedrig/niedrig $^{(\mathrm{d})}$ \\
\hline & Decressin und Decressin (2002) & SOEP & HLA & nein & niedrig \\
\hline & Fehr, Goette und Pfeiffer $(2002)^{(\mathrm{e})}$ & IABS & EFA & ja & hoch \\
\hline & Knoppik und Beissinger (2003) & IABS & EFA & ja & hoch/sehr hoch ${ }^{(d)}$ \\
\hline & Knoppik und Dittmar (2002) & SOEP & HLA & nein & 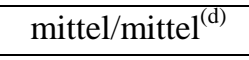 \\
\hline ITA & Devicienti (2003) & INPS & EFA & ja & hoch \\
\hline $\mathrm{CHE}$ & Fehr und Goette (2000) & SLFS & EFA & $\mathrm{ja}$ & sehr hoch \\
\hline CAN & Christofides und Leung (2003) & HRDC & HLA & $\mathrm{ja}$ & sehr hoch \\
\hline MEX & Castellanos (2001) & ENEU & HLA & nein & niedrig \\
\hline \multirow[t]{3}{*}{ USA } & Altonji und Devereux (2000) & PSID & EFA & ja & sehr hoch \\
\hline & Kahn (1997) & PSID & HLA & nein & mittel/niedrig $^{(\mathrm{d})}$ \\
\hline & Lebow, Saks und Wilson (1999) & ECI & HLA & nein & mittel \\
\hline
\end{tabular}

Anmerkungen:

Auswahl: Beschränkung auf Studien, die HLA (histogram-location approach) oder EFA (earningsfunction approach) verwenden. Sortierkriterien: Die Studien sind gemäß den folgenden Sortierkriterien angeordnet: 1. Ländergruppen (EU, Europa, Rest der Welt); 2. Länder, 3. Autoren.

(a) Daten: IABS (IAB Beschäftigten-Stichprobe), SOEP (Sozioökonomisches Panel), INPS (Istituto Nazionale della Previdenza Sociale), SLFS (Swiss Labor Force Survey), PSID (Panel Study of Income Dynamics), ECI (Employment Cost Index), HRDC (Human Resources Development Canada), ENEU (National Survey of Urban Employment).

(b) Messfehler Kontrolle: Die beiden Kategorien „Abwesenheit bzw. Modellierung von Messfehlern“ und „Vorhandensein und Nichtberücksichtigung von Messfehlern“ werden in der Tabelle als ,ja“/,nein“" vermerkt.

(c) Die Angaben zu den EFA-Studien stammen aus Knoppik und Beissinger (2002), Tab. 3, die Angaben für die HLA-Studien aus Knoppik und Dittmar (2002), Tab. 6.

(d) Arbeiter/Angestellte.

(e) Fehr, Goette und Pfeiffer (2002) analysieren nominelle und reale Rigidität; der Anteil derjenigen, deren gewünschte nominelle Kürzungen nicht umgesetzt werden können, ist hoch bis sehr hoch. 
Tabelle 3: Korrelation zwischen Einschätzung des Ausmaßes der Abwärtsnominallohnstarrheit und Arbeitsmarktregime

\begin{tabular}{|l|l|l|l|l|}
\hline \multicolumn{1}{|c|}{ Rigidität } & \multicolumn{1}{|c|}{ niedrig } & \multicolumn{1}{c|}{ mittel } & \multicolumn{1}{c|}{ hoch hoch } \\
\hline $\begin{array}{l}\text { angelsäch- } \\
\text { sisch }\end{array}$ & Kahn (1997) & $\begin{array}{l}\text { Lebow, Saks und } \\
\text { Wilson (1999) }\end{array}$ & & $\begin{array}{l}\text { Altonji und Devereux } \\
(2000), \\
\text { Christofides und } \\
\text { Leung (2003) }\end{array}$ \\
\hline $\begin{array}{l}\text { kontinental- } \\
\text { europäisch }\end{array}$ & $\begin{array}{l}\text { Beissinger und } \\
\text { Knoppik (2001), } \\
\text { Decressin und } \\
\text { Decressin (2002), }\end{array}$ & $\begin{array}{l}\text { Knoppik und Dittmar } \\
\text { (2002), }\end{array}$ & $\begin{array}{l}\text { Knoppik und } \\
\text { Beissinger (2003), } \\
\text { Devicienti (2003), } \\
\text { Fehr, Goette und } \\
\text { Pfeiffer (2002) }\end{array}$ & $\begin{array}{l}\text { Fehr und Goette } \\
\text { (2000), }\end{array}$ \\
\hline
\end{tabular}

Anmerkungen: Studien aus Tabelle 2 mit Ausnahme von Castellanos (2001) zu MEX. 
Tabelle 4: Korrelation zwischen Einschätzung des Ausmaßes der Abwärtsnominallohnstarrheit und Messfehlerkontrolle

\begin{tabular}{|l|l|l|l|l|}
\hline $\begin{array}{l}\text { Re } \\
\text { Kontrolle }\end{array}$ & \multicolumn{1}{|c|}{ niedrig } & \multicolumn{1}{|c|}{ hoch } & \multicolumn{1}{c|}{ sehr hoch } \\
\hline ja & & & $\begin{array}{l}\text { Knoppik und } \\
\text { Beissinger (2003), } \\
\text { Devicienti (2003), } \\
\text { Fehr, Goette und } \\
\text { Pfeiffer (2002) }\end{array}$ & $\begin{array}{l}\text { Altonji und Devereux } \\
(2000), \\
\text { Fehr und Goette } \\
(2000), \\
\text { Christofides und } \\
\text { Leung (2003) }\end{array}$ \\
\hline nein & $\begin{array}{l}\text { Beissinger und } \\
\text { Knoppik (2001), } \\
\text { Decressin und } \\
\text { Decressin (2002), } \\
\text { Kahn (1997), } \\
\text { Castellanos (2001) }\end{array}$ & $\begin{array}{l}\text { Knoppik und Dittmar } \\
\text { (2002), } \\
\text { Lebow, Saks und } \\
\text { Wilson (1999) }\end{array}$ & & \\
& & & \\
\end{tabular}

Anmerkungen: Studien aus Tabelle 2. 


\section{IZA Discussion Papers}

\begin{tabular}{|c|c|c|c|c|}
\hline No. & Author(s) & Title & Area & Date \\
\hline 786 & $\begin{array}{l}\text { P. Mueser } \\
\text { K. R. Troske } \\
\text { A. Gorislavsky }\end{array}$ & $\begin{array}{l}\text { Using State Administrative Data to Measure } \\
\text { Program Performance }\end{array}$ & 6 & $05 / 03$ \\
\hline 787 & $\begin{array}{l}\text { P. Garibaldi } \\
\text { L. Pacelli } \\
\text { A. Borgarello }\end{array}$ & $\begin{array}{l}\text { Employment Protection Legislation and the Size } \\
\text { of Firms }\end{array}$ & 3 & $05 / 03$ \\
\hline 788 & $\begin{array}{l}\text { F. Büchel } \\
\text { J. R. Frick }\end{array}$ & $\begin{array}{l}\text { Immigrants in the UK and in West Germany - } \\
\text { Relative Income Position, Income Portfolio, and } \\
\text { Redistribution Effects }\end{array}$ & 1 & $06 / 03$ \\
\hline 789 & $\begin{array}{l}\text { J. A. Cabral Vieira } \\
\text { A. R. Cardoso } \\
\text { M. Portela }\end{array}$ & $\begin{array}{l}\text { Recruitment and Pay at the Establishment } \\
\text { Level: Gender Segregation and the Wage Gap } \\
\text { in Portugal }\end{array}$ & 5 & $06 / 03$ \\
\hline 790 & $\begin{array}{l}\text { A. Cigno } \\
\text { A. Luporini } \\
\text { A. Pettini }\end{array}$ & $\begin{array}{l}\text { Hidden Information Problems in the Design of } \\
\text { Family Allowances }\end{array}$ & 3 & $06 / 03$ \\
\hline 791 & M. Güell & $\begin{array}{l}\text { Fixed-Term Contracts and the Duration } \\
\text { Distribution of Unemployment }\end{array}$ & 1 & $06 / 03$ \\
\hline 792 & $\begin{array}{l}\text { L. Borghans } \\
\text { B. ter Weel }\end{array}$ & $\begin{array}{l}\text { What Happens When Agent T Gets a } \\
\text { Computer? The Labor Market Impact of Cost } \\
\text { Efficient Computer Adoption }\end{array}$ & 5 & $06 / 03$ \\
\hline 793 & T. Palokangas & $\begin{array}{l}\text { Foreign Direct Investment, Labour Market } \\
\text { Regulation and Self-Interested Governments }\end{array}$ & 3 & $06 / 03$ \\
\hline 794 & $\begin{array}{l}\text { P. Frijters } \\
\text { M. A. Shields } \\
\text { S. Wheatley Price }\end{array}$ & $\begin{array}{l}\text { Investigating the Quitting Decision of Nurses: } \\
\text { Panel Data Evidence from the British National } \\
\text { Health Service }\end{array}$ & 1 & $06 / 03$ \\
\hline 795 & B. T. Hirsch & $\begin{array}{l}\text { Reconsidering Union Wage Effects: Surveying } \\
\text { New Evidence on an Old Topic }\end{array}$ & 3 & $06 / 03$ \\
\hline 796 & P. Apps & Gender, Time Use and Models of the Household & 5 & $06 / 03$ \\
\hline 797 & $\begin{array}{l}\text { E. Bratberg } \\
\varnothing . \text { A. Nilsen } \\
\text { K. Vaage }\end{array}$ & $\begin{array}{l}\text { Assessing Changes in Intergenerational } \\
\text { Earnings Mobility }\end{array}$ & 2 & $06 / 03$ \\
\hline 798 & $\begin{array}{l}\text { J. J. Heckman } \\
\text { J. A. Smith }\end{array}$ & $\begin{array}{l}\text { The Determinants of Participation in a Social } \\
\text { Program: Evidence from a Prototypical Job } \\
\text { Training Program }\end{array}$ & 6 & $06 / 03$ \\
\hline 799 & R. A. Hart & $\begin{array}{l}\text { General Human Capital and Employment } \\
\text { Adjustment in the Great Depression: } \\
\text { Apprentices and Journeymen in UK Engineering }\end{array}$ & 2 & $06 / 03$ \\
\hline 800 & $\begin{array}{l}\text { T. Beissinger } \\
\text { C. Knoppik }\end{array}$ & $\begin{array}{l}\text { Sind Nominallöhne starr? Neuere Evidenz und } \\
\text { wirtschaftspolitische Implikationen }\end{array}$ & 7 & $06 / 03$ \\
\hline
\end{tabular}

An updated list of IZA Discussion Papers is available on the center's homepage www.iza.org. 